\title{
O Estilo Motivacional do Professor e a Motivação Intrínseca dos Estudantes: Uma Perspectiva da Teoria da Autodeterminação
}

\author{
Sueli Édi Rufini Guimarães ${ }^{123}$ \\ Universidade Estadual de Londrina \\ Evely Boruchovitch \\ Universidade Estadual de Campinas
}

\begin{abstract}
Resumo
A Teoria da Autodeterminação foi proposta com o objetivo de compreender os componentes da motivação intrínseca e extrínseca e os fatores relacionados com a sua promoção. Nessa perspectiva, são abordadas a personalidade e a motivação humana, concentrando-se nas tendências evolutivas, nas necessidades psicológicas inatas e nas condições contextuais favoráveis à motivação, ao funcionamento social e ao bem estar pessoal. No contexto da pesquisa educacional, a motivação intrínseca tem sido relacionada ao envolvimento dos alunos com as tarefas de aprendizagem, pela preferência por desafios, persistência, esforço, uso de estratégias de aprendizagem, entre outros resultados positivos. Partindo da Teoria da Autodeterminação, este artigo tem como objetivos analisar os conceitos relativos à motivação intrínseca, postulados nessa teoria, refletir sobre o papel do professor, mas especificamente sobre seu estilo na promoção desse padrão motivacional no aluno, bem como discutir as implicações educacionais deste tema.

Palavras-chave: Motivação intrínseca; teoria da autodeterminação; estilo motivacional do professor.
\end{abstract}

Teacher's Motivational Style and Students' Intrinsic Motivation: The Self-determination Perspective

\begin{abstract}
The Self-determination Theory has the purpose of understanding the intrinsic and extrinsic components of motivation, and the factors that contribute for its promotion. In this perspective personality and human motivation are considered in terms of developmental tendencies, innate psychological needs and contextual variables which favour motivation, social functioning and personal well-being. In the context of educational research, intrinsic motivation has been characterized by students' involvement in learning tasks due to their preference for challenges, persistence, effort, as well as by their use of learning strategies. In line with that, the objectives of this paper are not only present and analyze the concepts related to intrinsic motivation according to the Self-determination Theory, but also reflect upon the teacher's role and style in the promotion of students' intrinsic motivation. Educational implications are also discussed.

Keywords: Intrinsic motivation; self-determination theory; teacher' motivational style.
\end{abstract}

A escola representa para a sociedade ocidental uma fonte socializadora de grande impacto na vida das pessoas. Para alcançar seus objetivos é necessário, no entanto, que se promova entre os estudantes interesse genuíno e entusiasmo pela aprendizagem e desempenho escolar (Pajares \& Schunk, 2001).

A motivação no contexto escolar tem sido avaliada como um determinante crítico do nível e da qualidade da aprendizagem e do desempenho. Um estudante motivado mostra-se ativamente envolvido no processo de aprendizagem, engajando-se e persistindo em tarefas desafiadoras, despendendo esforços, usando estratégias adequadas, buscando desenvolver novas habilidades de compreensão e de domínio. Apresenta entusiasmo na execução das tarefas e orgulho acerca dos resultados de

\footnotetext{
${ }^{1}$ Apoio financeiro CAPES - Bolsa de capacitação.

${ }^{2}$ Endereço para correspondência: Rua Rangel Pestana, 340, 1301, 86062 020, Jardim Campo Belo, Londrina, Paraná. E-mail: jsgj@uel.br

${ }^{3}$ Trabalho parte da tese de doutorado da primeira autora, sob orientação da segunda autora, no Programa de Doutorado em Educação da UNICAMP.
}

seus desempenhos, podendo superar previsões baseadas em suas habilidades ou conhecimentos prévios.

A motivação intrínseca é o fenômeno que melhor representa o potencial positivo da natureza humana, sendo considerada por Deci e Ryan (2000), Ryan e Deci (2000a), entre outros, a base para o crescimento, integridade psicológica e coesão social. Configura-se como uma tendência natural para buscar novidade, desafio, para obter e exercitar as próprias capacidades. Refere-se ao envolvimento em determinada atividade por sua própria causa, por esta ser interessante, envolvente ou, de alguma forma, geradora de satisfação. Tal envolvimento é considerado ao mesmo tempo espontâneo, parte do interesse individual, e autotélico (Csikszentmihalyi, 1992), isto é, a atividade é um fim em si mesma.

As descobertas sobre as orientações motivacionais autodeterminadas, ou seja, a motivação intrínseca e as formas auto-reguladas de motivação extrínseca, representam alternativas promissoras para se alcançar o envolvimento dos estudantes com a escola e com sua própria educação 
(Deci, Vallerand, Pelletier \& Ryan, 1991). Procurando compreender os determinantes motivacionais e descobrir contextos promotores das formas autodeterminadas de motivação, foi desenvolvida a Teoria da Autodeterminação. Assim sendo, são objetivos deste artigo introduzir e analisar os conceitos e os determinantes relacionados à motivação intrínseca, usando como referencial teórico a Teoria da Autodeterminação. Tem-se em vista refletir sobre o papel do professor na promoção da motivação intrínseca, bem como discutir as implicações educacionais deste tema que, embora apontado como relevante pela literatura internacional (Amabile, Hill, Hennessey \& Tighe, 1994; Andersen, Chen \& Carter, 2000; Cai, Reeve \& Robinson, 2002, Guay, Boggiano \& Vallerand, 2001; Pelletier, Sèguin-Levesque \& Legault, 2002; Ryan \& Deci 2000a, 2000b; entre outros), ainda é pouco difundido no Brasil .

\section{Uma Introdução à Teoria da Autodeterminação}

Segundo revisão realizada por Deci e Ryan (2000), nos anos 1970, principalmente nos Estados Unidos da América, ainda era evidente a influência das abordagens comportamentais na psicologia empírica. Influenciados pelas proposições de White (1975) a respeito do envolvimento das pessoas em atividades apenas pela busca de eficácia ou de competência, como também pelas idéias de deCharms (1984) sobre a propensão natural humana para ser agente causal das próprias ações, alguns pesquisadores iniciaram a exploração do conceito de motivação intrínseca. Em 1975, Deci (citado em Deci \& Ryan, 2000) apresenta a organização dessas concepções teóricas no livro intitulado Intrinsic Motivation afirmando que, para serem intrinsecamente motivadas, as pessoas necessitariam se sentir competentes e autodeterminadas. Em sua argumentação, contrapôs as afirmações de Skinner (1998) acerca da ligação funcional entre comportamento e reforçamento, reiterando que os comportamentos intrinsecamente motivados seriam independentes de conseqüências operacionalmente separadas porque, nesse caso, a realização da atividade seria a própria recompensa. Além disso, nesse trabalho, propôs o conceito de necessidades psicológicas básicas, apontadas como determinantes do comportamento intrinsecamente motivado, contrariando idéias anteriores de que todo comportamento seria função da satisfação de necessidades fisiológicas.

Segundo Deci e Ryan (2000), centenas de estudos foram desenvolvidos a partir dessa etapa sendo que, em sua maioria, buscavam comparar a motivação intrínseca com a motivação extrínseca. Os resultados dessas investigações indicavam que as recompensas materiais prejudicariam a motivação intrínseca, reduzindo o envolvimento na atividade para níveis menores do que os apresentados antes da introdução das recompensas. As explicações para o problema tomavam como base a idéia de mudança na percepção do locus de causalidade (deCharms, 1984), isto é, as pessoas deixavam de perceber suas ações como internamente guiadas para se sentirem externamente comandadas.

Com o incremento de trabalhos empíricos, voltados para a compreensão do fenômeno e o amadurecimento teórico atingido, Deci e colaboradores (Deci \& Ryan, 1985; Ryan, Connell \& Deci, 1985) desenvolveram a Teoria da Autodeterminação, abordando a personalidade e a motivação humanas, focalizando as tendências evolutivas, as necessidades psicológicas inatas (consideradas como a base para a motivação e integração da personalidade) e as condições contextuais favoráveis à motivação, ao funcionamento social e ao bem-estar pessoal. Estes autores utilizaram, preferencialmente, metodologias experimentais em suas investigações, incluindo a manipulação direta de variáveis contextuais e o subseqüente exame dos seus efeitos sobre os processamentos internos e manifestações comportamentais. Como resultados das investigações, são identificados, atualmente, diferentes tipos de motivação como a regulação intencional, a autodeterminação e o controle externo, tendo cada um deles conseqüências específicas sobre aprendizagem, desempenho, experiência e bem-estar pessoal. Segundo avaliação de Andersen e colaboradores (2000), o modelo proposto pela Teoria da Autodeterminação tem revolucionado os estudos sobre motivação intrínseca e extrínseca nas duas últimas décadas.

A base inicial para a Teoria da Autodeterminação é a concepção do ser humano como organismo ativo, dirigido para o crescimento, desenvolvimento integrado do sentido do self e para integração com as estruturas sociais. Nesse empenho evolutivo estaria incluída a busca de experiências com atividades interessantes para alcançar os objetivos de: a) desenvolver habilidades e exercitar capacidades; b) buscar e obter vínculos sociais; e c) obter um sentido unificado do self por meio da integração das experiências intrapsíquicas e interpessoais. Nessa perspectiva, consideram-se as ações autodeterminadas como essencialmente voluntárias e endossadas pessoalmente e, em contraposição, as ações controladas como resultado de pressões decorrentes de forças interpessoais ou intrapsíquicas. No primeiro caso, a regulação do comportamento é escolhida pela pessoa, já no segundo caso, o processo de regulação pode ou não ser consentido. Aplicada ao contexto educacional, a Teoria da Autodeterminação focaliza a promoção do interesse dos estudantes pela aprendizagem, a valorização da educação e a confiança nas próprias capacidades e atributos.

Com o objetivo de compreender a energia e a direção do comportamento motivado, a Teoria da Autodeterminação postula a existência de algumas necessidades psicológicas básicas e inatas que movem os seres humanos, sendo 
definidas como os nutrientes necessários para um relacionamento efetivo e saudável destes com seu ambiente. Uma vez satisfeita, a necessidade psicológica promove sensação de bem-estar e de um efetivo funcionamento do organismo (Deci \& Ryan, 1985, 1996, 2000; Deci \& cols., 1991; Ryan \& Deci, 2000a, 2000b).

Segundo Deci e Ryan (2000), o emprego do conceito de necessidades favorece a descoberta de universais motivacionais para as ações humanas que, por sua vez, representariam um substrato do qual poderiam ser extraídos e integrados fenômenos que, de um modo mais superficial, pareceriam sem vinculação. Além disso, o conhecimento das condições de satisfação das necessidades psicológicas básicas indicariam as características dos contextos facilitadores da motivação, desenvolvimento e desempenho.

Nesta perspectiva teórica, a atenção para as necessidades sócio-emocionais dos estudantes é essencial para a construção de um ambiente educacional potencialmente motivador, principalmente por parte de professores e administradores escolares. Assim, como um ponto de partida para essa tarefa, é preciso conhecer os conceitos e o que a pesquisa empírica tem descoberto sobre o tema.

\section{A Teoria da Autodeterminação e as Necessidades de Autonomia, Competência e de Vínculo}

Três necessidades psicológicas inatas, subjacentes à motivação intrínseca, são propostas pela Teoria da Autodeterminação: a necessidade de autonomia, a necessidade de competência e a necessidade de pertencer ou de estabelecer vínculos. A satisfação das três é considerada essencial para um ótimo desenvolvimento e saúde psicológica. Em situações de aprendizagem escolar, as interações em sala de aula e na escola como um todo precisam ser fonte de satisfação dessas três necessidades psicológicas básicas para que a motivação intrínseca e as formas autodeterminadas de motivação extrínseca possam ocorrer. Nesse sentido, a figura do professor tem um papel essencial na promoção de um clima de sala de aula favorável ou não ao desenvolvimento dessas orientações motivacionais.

Autonomia significa a faculdade de se governar por si mesmo; o direito ou faculdade de se reger (uma nação) por leis próprias; liberdade ou independência moral ou intelectual. $\mathrm{O}$ adjetivo autônomo refere-se a agir sem controle externo (Ferreira, 1986). Para a Teoria da Autodeterminação, o conceito de autonomia é vinculado ao desejo ou a vontade do organismo de organizar a experiência e o próprio comportamento e para integrá-los ao sentido do self.

A idéia de necessidade psicológica básica de autodeterminação ou autonomia foi inspirada no trabalho de deCharms (1984) que, estendendo um conceito introduzido por Heider (1958, citado em Deci \& cols., 1985) sobre a causação pessoal, destacou a autodeterminação como sendo uma necessidade humana inata, relacionada à motivação intrínseca. Segundo essa perspectiva, as pessoas seriam naturalmente propensas a realizar uma atividade por acreditarem que o fazem por vontade própria, porque assim o desejam e não por serem obrigados devido a demandas externas sendo, nesse caso, denominadas "origem" ou como tendo locus de causalidade interno.

O indivíduo "origem" tem fortes sentimentos de causação pessoal e atribui as mudanças produzidas em seu contexto às suas próprias ações. Em decorrência dessa percepção, apresenta comportamento intrinsecamente motivado, fixa metas pessoais, demonstra seus acertos e dificuldades, planeja as ações necessárias para viabilizar seus objetivos e avalia adequadamente seu progresso.

Em contrapartida, o locus de causalidade externo implica em outro agente ou objeto, interferindo com a causação pessoal, levando a pessoa a se perceber como um "marionete", resultando em sentimentos negativos de ser externamente guiado. $\mathrm{O}$ indivíduo com essa percepção acredita que as causas de seus comportamentos estão relacionadas a fatores externos, como o comportamento ou a pressão de outras pessoas. Perceber-se como externamente guiado promove sentimentos de fraqueza e ineficácia, implicando no afastamento de situações de desempenho, acarretando o desenvolvimento precário daquelas habilidades que possibilitariam uma melhor interação com eventos do ambiente. Isto ocorre porque, ao sentir-se obrigado por fatores externos a realizar algo, o indivíduo tem sua atenção desviada da tarefa, prejudicando assim a motivação intrínseca. Desse modo, o conceito de autonomia, segundo a Teoria da Autodeterminação, é vinculado ao desejo ou a vontade pessoal de organizar a experiência e o próprio comportamento e integrá-los ao sentido do self.

Ryan e Deci (2000a, 2000b) reconhecem que o conceito de autonomia não tem recebido ampla aceitação pelos teóricos da área, mesmo estando apoiado em resultados de diversos estudos empíricos. Enquanto a necessidade de competência e de pertencer ou estabelecer vínculos são objetos de investigação de diversos autores, em várias linhas teóricas da psicologia, a Teoria da Autodeterminação está praticamente sozinha na exploração do constructo de necessidade básica de autonomia.

Alguns autores têm questionado, por exemplo, se a autonomia é realmente uma necessidade ou é apenas um produto da ideologia ocidental (Carver \& Scheier, 2000). Para rebater tais críticas, Ryan e Deci (2000a, 2000b) afirmam que elas são embasadas em definições de autonomia que não coincidem com aquelas teorizadas e operacionalizadas pela Teoria da Autodeterminação. Em sua maioria, os críticos falam de autonomia ligada a idéias de independência, 
individualismo ou desapego e, bem diferente dessas definições, a necessidade de autonomia proposta pela Teoria da Autodeterminação tem como elementos centrais a vontade e a auto-regulação integradora. Em suma, autonomia aqui significa auto-governo, auto-direção, autodeterminação.

Seria inconcebível imaginar, argumentam Deci e Ryan (2000), que houvesse alguma situação em nossa vida cotidiana na qual pudéssemos agir de modo totalmente independente das influências externas. O cerne da questão está no fato da pessoa contribuir com as forças que influenciam suas ações, ou seja, se ela permanece de modo passivo diante das de demandas externas, um "marionete" na concepção de deCharms (1984) ou, ao contrário, as aceita, compreende-as por seu valor e utilidade, percebendo-as como fonte de informações que servem de apoio para as suas iniciativas.

A proposta de uma necessidade de competência como fator determinante da motivação intrínseca foi baseada nos trabalhos de White (1975) que utilizou o termo competência para definir a capacidade do organismo de interagir satisfatoriamente com o seu meio. No final da década de 1950, White publicou um artigo que se tornou clássico, destacando como força motivacional inata a necessidade do ser humano agir de modo eficaz em seu ambiente. Os estudos de White (1975) foram influenciados pelo descontentamento com as explicações provenientes da teoria do drive que, segundo revisão de Weiner (1990) e Graham e Weiner (1996), teria sido a abordagem teórica que mais influenciou os trabalhos experimentais durante as décadas de 1950 a 1970.

De acordo com a concepção de White (1975), em virtude das poucas aptidões inatas dos seres humanos para um nível eficiente de interações com o meio, faz-se necessário que aprendam e desenvolvam as capacidades exigidas. Vista desse modo, a competência teria um aspecto motivacional que orientaria o organismo a tentativas de domínio, não podendo ser atribuída a impulsos frente a necessidades específicas ou a instintos. Esta necessidade de relacionamento eficaz foi considerada intrínseca, isto é, a gratificação proporcionada seria inerente à própria interação. A experiência de dominar uma tarefa desafiadora e o aumento da competência dela resultante trazem emoções positivas, as quais White (1975) denominou "sentimento de eficácia".

A Teoria da Autodeterminação afirma que os eventos sócio-contextuais que fortalecem a percepção de competência no decorrer de uma ação, por exemplo, ofeedback positivo em situações de desafio de nível ótimo, aumentam a ocorrência da motivação intrínseca. No entanto, somente o sentimento de competência não é suficiente para promover um aumento da motivação intrínseca. É necessário que seja acompanhado por uma percepção de autonomia, ou seja, a situação não deve sufocar o senso de liberdade individual, como também a pessoa precisa se sentir responsável pelo desempenho competente. Desse modo, parece que as circunstâncias que promovem a percepção de autonomia e de competência, denominadas informativas, são promotoras da motivação intrínseca.

As necessidades psicológicas básicas de competência e de autonomia têm sido consideradas essenciais para a motivação intrínseca. No entanto, resultados de pesquisas apontam para uma terceira necessidade: a de pertencer ou de estabelecer vínculos. No atual estágio de desenvolvimento da área, essa necessidade é considerada menos central na determinação da motivação intrínseca, comparada às necessidades de competência e de autonomia. Isto se deve ao fato de que grande parte das atividades intrinsecamente motivadas são realizadas isoladamente, por isso ela é vista como um "pano de fundo", uma sensação de segurança que possibilita o desenvolvimento dessa tendência inata para $\mathrm{o}$ crescimento saudável.

Segundo Reeve e Sickenius (1994), Harlow, nos anos 1950, já apontava que, para um desenvolvimento adequado, as pessoas necessitariam se sentir amadas e de manter contato interpessoal, compondo uma base segura que sustentaria o ímpeto de exploração para os indivíduos em qualquer fase da vida. Embora os estudos sobre este tema tenham se desenvolvido principalmente focalizando a interação entre pais e filhos pequenos, trabalhos envolvendo interação professor/aluno confirmam a relevância de se promover em sala de aula um contexto de relação segura, no qual o professor demonstraria interesse e disponibilidade para atender as necessidades e perspectivas dos alunos.

Conceitualmente, a necessidade de pertencer seria uma tendência para estabelecer vínculo emocional ou para estar emocionalmente ligado e envolvido com pessoas significativas. Baumeister e Leary (1995) a definem como uma necessidade universal, aplicável a uma ampla diversidade de situações, sendo fonte de influência para padrões emocionais e cognitivos. Nessa perspectiva, todas as pessoas seriam compelidas a estabelecer e manter, pelo menos em quantidade mínima, relacionamentos interpessoais positivos, duradouros e significativos. Quando essa necessidade é frustrada, ao menos em parte, suas conseqüências afetam o equilíbrio emocional e o bem-estar geral do indivíduo.

Para Osterman (2000), a percepção de segurança nos relacionamentos dos estudantes com pais, professores e colegas é associada à autonomia, ao controle interno, ao bom relacionamento com figuras de autoridade e a níveis adequados de ansiedade. Ao contrário, sentimentos de insegurança nos mesmos relacionamentos são vinculados ao baixo auto-conceito, à incapacidade de agir de modo independente e à dificuldade ou incapacidade de se conformar com as normas. Os resultados das investigações indicam que alunos seguros em relação a seus pais e 
professores aceitam de forma mais positiva os fracassos acadêmicos, são mais autônomos, mais envolvidos com a aprendizagem e se sentem melhor a respeito de si mesmos.

De acordo com a revisão de Baumeister e Leary (1995), os estudantes que se sentem aceitos em seus diferentes relacionamentos desenvolvem uma orientação positiva em relação à escola, aos trabalhos e atividades escolares e aos professores. Crianças consideradas rejeitadas pelos colegas percebem a escola de modo significativamente desfavorável, faltam muito às aulas e apresentam níveis de desempenho mais baixos, comparadas às outras crianças mais integradas. Enfatizam os autores que a rejeição é relacionada a várias modalidades de estresse emocional, incluindo a solidão, a violência e o suicídio. Além disso, os comportamentos agressivos dos alunos que se sentem rejeitados na escola são os recursos que eles têm para tentar estabelecer ou manter relações com os demais membros daquele grupo. Lamentavelmente, além de frustradas suas tentativas, os seus relacionamentos tornam-se ainda mais prejudicados.

Um aspecto particularmente importante, destacado por Osterman (2000), refere-se ao apoio oferecido pelos professores. Comparado ao apoio por parte da família e dos colegas, o oferecido pelo professor tem uma influência direta sobre o envolvimento dos alunos com a escola e com as atividades escolares. Segundo o autor, o papel do professor deveria ser cuidadosamente analisado, possibilitando uma compreensão mais adequada dos motivos comumente atribuídos à falta de motivação ou de atitudes impróprias dos estudantes em relação à escola. Geralmente, os problemas neste âmbito são associados a causas internas, particulares do aluno, ao seu ambiente familiar ou ao próprio grupo de colegas a que pertence.

A ligação entre as necessidades psicológicas básicas de pertencer ou estabelecer vínculos e de autonomia, como determinantes da motivação intrínseca pode, à primeira vista, parecer inconsistente. No entanto, Ryan e Stiller (1991) argumentam que ser autônomo não implica em ser desvinculado das outras pessoas, mas depende da percepção de ser agente e autodeterminado. Além disso, a autonomia tem seu pleno desenvolvimento naquelas situações em que crianças e adolescentes podem se sentir vinculados a adultos significativos.

As três necessidades psicológicas básicas, de competência, autonomia e vínculo, são integradas e interdependentes. Desse modo, a satisfação de cada uma delas reforça e fortalece as demais (Deci \& Ryan, 2000). Conseqüentemente, os contextos sociais facilitadores da motivação intrínseca têm em comum interações que consideram as necessidades de seus membros e são zelosos em suprí-las.

$\mathrm{Na}$ escola, a qualidade do relacionamento entre professores e alunos, influenciada em grande medida pelo estilo motivacional dos primeiros, revela-se a fonte principal de satisfação ou frustração das necessidades psicológicas dos estudantes. Tendo em vista a relevância do estilo motivacional do professor na promoção da motivação intrínseca dos alunos, a seguir este constructo será definido e serão apresentados estudos que fornecem subsídios para uma melhor compreensão dessa relação.

\section{O Estilo Motivacional do Professor e a Motivação Intrínseca do Aluno}

A motivação intrínseca do aluno não resulta de treino ou de instrução, mas pode ser influenciada principalmente pelas ações do professor. Embora não se desconsiderem as crenças, conhecimentos, expectativas e hábitos que os estudantes trazem para a escola, a respeito da aprendizagem e da motivação, o contexto instrucional imediato, ou seja, a sala de aula, torna-se fonte de influência para o seu nível de envolvimento (Ames, 1992; Givvin, Stipek, Salmon \& Macgyvers, 2001; Guthrie \& Alao, 1997; Stipek, 1998).

De acordo com Reeve (1998) e Reeve, Bolt e Cai (1999), o estilo motivacional refere-se à crença e confiança do professor em determinadas estratégias de ensino e de motivação. Algumas pessoas teriam personalidades mais voltadas para o controle, ou seja, personalidades autoritárias, enquanto outras tenderiam mais a respeitar o outro em suas interações. A possibilidade de apoiar a autonomia alheia seria fruto de um estilo interpessoal composto, em parte, por características de personalidade e por habilidades adquiridas. Estas habilidades, passíveis de aprendizagem, incluiriam tomar a perspectiva da outra pessoa, reconhecer seus sentimentos, usar linguagem não-controladora, oferecer informações importantes para tomadas de decisão, entre outras. O estilo motivacional do professor é considerado, portanto, uma característica vinculada à personalidade, mas é vulnerável a fatores sócio-contextuais como, por exemplo, o número de alunos em sala de aula, o tempo de experiência no magistério, o gênero, a idade, as interações com a direção da escola, as concepções ideológicas, entre outros. Além disso, a interação dos professores com seus alunos extrapola as disposições pessoais por englobar a sua percepção acerca do envolvimento dos estudantes, das pressões sofridas no decorrer do ano letivo, provenientes das relações com a comunidade, como pais e diretores e o tipo de avaliação do trabalho utilizado pela escola.

Os estudos desenvolvidos sobre o tema têm considerado dois estilos motivacionais do professor, propostos inicialmente por Deci, Schwartz, Sheinman e Ryan (1981), que variam em um continuum de altamente controlador a altamente promotor de autonomia. Os professores facilitadores da autonomia de seus alunos nutrem suas necessidades psicológicas básicas de autodeterminação, de competência e de segurança. Para que isso ocorra, eles 
oferecem oportunidade de escolhas e de feedback significativos, reconhecem e apóiam os interesses dos alunos, fortalecem sua auto-regulação autônoma e buscam alternativas para leválos a valorizar a educação, em suma, tornam o ambiente de sala de aula principalmente informativo. Apoiar a autonomia dos alunos significa, nessa perspectiva, incentivá-los a fazer escolhas, a participar das tomadas de decisão sobre sua educação e levá-los a se identificar com as metas de aprendizagem estabelecidas em sala de aula. É importante destacar que, para fortalecer a percepção de autonomia, as opções de escolha oferecidas aos alunos devem ser referentes a aspectos importantes do contexto de aprendizagem, que uma vez escolhidas tornem-se reais, que todas as informações sobre as opções sejam explicitadas, objetivando uma escolha segura (Guthrie \& Alao, 1997).

Em contrapartida, os professores que confiam em um estilo relativamente controlador estabelecem para seus alunos formas específicas de comportamentos, sentimentos ou de pensamentos, oferecendo incentivos extrínsecos e conseqüências para aqueles que se aproximam do padrão esperado. No ambiente de sala de aula o controle é a principal característica.

Não há dúvida de que o estilo motivacional do professor configura-se em uma importante fonte de influência para o desempenho, emoções e motivação dos alunos em relação à escola. Pesquisas têm demonstrado ser este um fator relativamente estável durante o ano letivo. Patrick, Anderman, Ryan, Edelin e Midgley (2001), em um estudo empregando metodologias qualitativas e quantitativas, descobriram que as normas, as interações e as práticas de ensino, apresentadas no início do ano letivo, não tiveram grandes modificações no decorrer do período, mas foram fonte de influência para as percepções dos alunos sobre as estruturas de meta presentes em sala de aula.

As estruturas de meta referem-se aos objetivos assinalados e aos padrões comportamentais valorizados em sala de aula, transmitidas aos alunos de modo implícito ou explícito por meio das diversas ações do professor como, por exemplo, as características das atividades solicitadas, as formas de avaliação, de reconhecimento dos interesses e necessidades dos estudantes, os critérios para formação de grupos, o uso do tempo e o modo como o professor compartilha a autoridade. Tais estruturas influenciam as metas adotadas pelos alunos em relação à escola, aos trabalhos escolares e, de modo geral, em relação a sua educação. Para um maior aprofundamento no assunto, as metas de realização são apresentadas no trabalho de Bzuneck (2001) e a organização da escola e da sala de aula visando a motivação dos estudantes é descrita em Guimarães (2001).
Resultados de pesquisas, realizadas com alunos desde o ensino fundamental até o nível universitário, indicam que alunos de professores com estilo motivacional promotor de autonomia demonstram maior percepção de competência acadêmica, maior compreensão conceitual, melhor desempenho, perseveram na escola, aumentam sua criatividade para as atividades escolares, buscam desafios, são emocionalmente mais positivos, menos ansiosos, buscam o domínio e são mais intrinsecamente motivados, quando comparados a alunos de professores com estilo motivacional controlador (Cai \& cols., 2002; Deci \& cols., 1981; Deci, Spiegel, Ryan, Koestner \& Kauffman, 1982; Flink, Boggiano \& Barret, 1990; Guay \& cols., 2001; Patrick \& cols., 2000; Patrick, Hisley \& Kempler, 2001; Pelletier \& cols. 2002; Skinner \& Belmont, 1993; Vallerand, Fortier \& Guay, 1997; Williams \& Deci, 1996).

Em suma, o estilo motivacional do professor é importante fonte de influência para a orientação motivacional dos estudantes, refletindo no seu desempenho escolar e, por isso, merece interesse e atenção por parte dos pesquisadores.

\section{Considerações Finais}

A literatura sobre a motivação no contexto escolar tem destacado os resultados positivos para aprendizagem e desempenho dos alunos decorrentes da orientação motivacional intrínseca. Para sua promoção, de acordo com a Teoria da Autodeterminação, é essencial a satisfação das necessidades psicológicas básicas de autonomia, competência e vínculo. Nesse aspecto, o estilo motivacional do professor revela-se um importante constructo educacional pelo impacto que exerce no desenvolvimento motivacional dos estudantes.

Como proposta recente, apesar de apoiada em resultados de inúmeros trabalhos empíricos, muito ainda está para ser descoberto e aprofundado com base na Teoria da Autodeterminação. Especificamente para a realidade educacional brasileira, um grande percurso precisa ser trilhado, pois o interesse dos pesquisadores pelo tema motivação ainda é incipiente em nossa literatura. Mesmo assim, com base nos relatos de investigações realizadas em outras culturas, pode-se pressupor que as implicações educacionais dos trabalhos nessa área são relevantes, já que oferecem alternativas para as interações em sala de aula, visando a melhoria do envolvimento dos estudantes para com a aprendizagem escolar.

De acordo com o atual desenvolvimento da área, as expectativas dos professores sobre como envolver os alunos com as atividades escolares podem ser concretizadas, em parte, na medida em que o ambiente escolar supra as 
necessidades psicológicas básicas dos estudantes. Esta não é uma tarefa de fácil realização, mas depende do esforço em se criar interações promotoras de autonomia e menos controladoras, por exemplo, dando oportunidades de escolha e tornando os alunos responsáveis pelas conseqüências de suas opções, compartilhando as tomadas de decisão, ouvindo e questionando os argumentos dos estudantes, entre outras estratégias. A necessidade de competência pode ser nutrida por meio da apresentação de desafios adequados para o nível de desenvolvimento dos alunos e com o retorno de informações sobre o seu desempenho. Finalmente, a criação da cultura de uma "comunidade escolar" na qual todos os alunos e professores sintam-se aceitos e emocionalmente vinculados representaria o chamado "pano de fundo", ou seja, a segurança necessária para a ação de aprender.

\section{Referências}

Amabile, T. M., Hill, K. G., Hennessey, B. A. \& Tighe, E. M. (1994). The work preference inventory assessing intrinsic and extrinsic motivational orientations. Journal of Personality and Social Psychology, 6(5), 950-967.

Ames, C. (1992). Classroom: Goals, structures, and student motivation. Journal of Educational Psychology, 84(3), 261-271.

Andersen, S. M., Chen, S. \& Carter, C. (2000). Fundamental human needs: Making social cognition relevant. Psychological Inquiry, 11(4), 269-318.

Baumeister, R. F. \& Leary, M. R. (1995). The need to belong: Desire for interpersonal attachments as a fundamental human motivation. Psychological Bulletin, 117(3), 497-529.

Bzuneck, J. A. (2001). A motivação do aluno orientado a metas de realização. Em E. Boruchovith \& J. A. Bzuneck (Orgs.), A motivação do aluno: Contribuições da psicologia contemporânea (pp. 58-77). Petrópolis, RJ: Vozes.

Cai, Y., Reeve, J. \& Robinson, D. T. (2002). Home schooling and teaching style: Comparing the motivating styles of home school and public school teachers. Journal of Educational Psychology, 94(2), 372-380.

Carver, C. S. \& Scheier, M. F. (2000). Autonomy and self-regulation. Psychological Inquiry, 11(4), 227-268.

Csikszentmihalyi, M. (1992). A psicologia da felicidade. São Paulo: Saraiva.

deCharms, R. (1984). Motivation enhancement in educational settings. Em C. Ames \& R. Ames (Orgs.), Research on motivation in education, student motivation (pp. 275-310). New York: Academic Press.

Deci, E. L. \& Ryan, R. M. (1985). Intrinsic motivation and self-determination in buman behavior. New York: Plennum Press.

Deci, E. L. \& Ryan, R. M. (1996). Need satisfaction and the self-regulation of learning. Learning \& Individual Differences, 18(3), 165-184.

Deci, E. L. \& Ryan, R. M. (2000). The "what" and "why" of goal pursuits: Human needs and self-determination of behavior. Psychological Inquiry, 11(4), 227-268.

Deci, E. L., Spiegel, N. H., Ryan, R. M., Koestner, R. \& Kauffman, M. (1982). Effects of performance standards on teaching styles: Behavior of controlling teachers. Journal of Educational Psychology, 74(6), 852-859.

Deci, E. L., Schwartz, A. J., Sheinman, L. \& Ryan, R. M. (1981). An instrument to assess adults'orientations toward control versus autonomy with children: Reflections on intrinsic motivation and perceived competence. Journal of Educational Psychology, 73(5), 642-650.
Deci, E. L., Vallerand, R. J., Pelletier, L. G. \& Ryan, R. M. (1991). Motivation in education: The self-determination perspective. Educational Psychologist, 26(3/ 4), 325-346.

Ferreira, A. B. H. (1986). Novo dicionário da língua portuguesa. Rio de Janeiro: Nova Fronteira.

Flink, C., Boggiano, A. K. \& Barret, M. (1990). Controlling teaching strategies: Undermining children's self-determination and performance. Journal of Personality and Social Psychology, 59(5), 916-924.

Givvin, K. B., Stipek, D. J., Salmon, J. M. \& Macgyvers, V. L. (2001). In the eyes of the beholder: Students' and teachers' judgments of students' motivation. Teacbing and Teacher Education, 17, 321-331.

Graham, S. \& Weiner, B. (1996). Theories and principles of motivation. Em D. C. Berliner \& R. C. Calfee (Orgs), Handbook of educational psychology (pp. 6384). New York: Simon \& Schuster Macmillan.

Guay, F., Boggiano, A. K. \& Vallerand, R. J. (2001). Autonomy support, intrinsic motivation, and perceived competence: Conceptual and empirical linkages. Personality and Social Psychology Bulletin, 27(6), 643-650.

Guimarães, S. É. R. (2001). A organização da escola e da sala de aula como determinate da motivação intrínseca e da meta aprender. Em E. Boruchovith \& J. A. Bzuneck (Orgs.), A motivação do aluno: Contribuições da psicologia contemporânea (pp. 78-95). Petrópolis, RJ: Vozes.

Guthrie, J. T. \& Alao, S. (1997). Designing contexts to increase motivations for reading. Educational Psychologist, 32(2), 95-105.

Osterman, K. F (2000). Students' need for belonging in the school community. Review of Educational Research, 70(3), 323-367.

Pajares, F. \& Schunk, D. H. (2001). Self-beliefs and school success: Self-efficacy, self-concept, and school achievement. Em R. Riding \& S. Rayner (Orgs.), Perception (pp. 239-266). London: Ablex.

Patrick, H., Anderman, L. H., Ryan, A. M. L., Edelin, K. C. \& Midgley, C. (2001). Teachers' comunication of goal orientations in four fifth-grade classrooms. The Elementary School Journal, 102(1), 35-59.

Patrick, H., Hisley, J. \& Kempler, T. (2000). “What's everybody so excited about?”: The effects of teacher entusiasm on student intrinsic motivation and vitality. The Journal of Experimental Education, 68(3), 217-236.

Pelletier, L. G., Sèguin-Levesque, C. \& Legault, L. (2002). Pressure from above and pressure from below as determinants of teachers' motivation and teaching behaviors. Journal of Educational Psychology, 94(1), 186-196.

Reeve, J. (1998). Autonomy support as na interpessoal motivating style: Is it teachable? Contemporary Educational Psychology, 23, 312-330.

Reeve, J., Bolt, E. \& Cai, Yi (1999). Autonomy-supportive teachers: How they teach and motivate students. Journal of Educational Psychology, 3, 1-12.

Reeve, J. \& Sickenius, B. (1994). Development and validation of a brief measure of three psychological needs underlyng intrinsic motivation: The AFS scales. Educational \& Psychological Measurement, 54(2), 506-516.

Ryan, R. M., Connell, J. P. \& Deci, E. L. (1985). A motivational analysis of selfdetermination and self-regulation in education. Em C. Ames \& R. Ames (Orgs.), Research on motivation in education (pp. 16-31). New York: Academic Press.

Ryan, R. M. \& Deci, E. L. (2000a). The darker and brighter sides of human existence: Basic psychological needs as a unifying concept. Psychological Inquiry, 11(4), 319-338.

Ryan, R. M. \& Deci, E. L. (2000b). Self-determination theory and the facilitation of intrinsic motivation, social development, and well-being. American Psychologist, 55(1), 68-78.

Ryan, R. M. \& Stiller, J. (1991). The social contexts of internalization: Parent and teacher influences on autonomy, motivation, and learning. Em C. Ames \& R. Ames (Orgs.), Advances in motivation and achievement (pp. 115-149). Connecticut: Jai.

Skinner, B. F. (1998). Ciência e comportamento bumano. São Paulo: Martins Fontes. 
Skinner, E. A \& Belmont, M. J. (1993). Motivation in the classroom: Reciprocal effects of teacher behavior and student engagement across the school year. Journal of Educational Psychology, 85(4), 571-581.

Stipek, D. J. (1998). Motivation to learn: From theory to pratice. Englewood Cliffs: Prentice-Hall.

Vallerand, R. J., Fortier, M. S. \& Guay, F. (1997). Self-determination and persistence in a real-life setting: Toward a motivational model of high school dropout. Journal of Personality and Social Psychology, 72(5), 1161-1176.

Weiner, B. (1990). History of motivational research in education. Journal of Educational Psychology, 82(4), 616-622.
White, W. R. (1975). Motivation reconsidered: The concept of competence. Em P. H. Mussem, J. J. Conger \& J. Kagan (Orgs.), Basic and contemporary issues in developmental psychology (pp. 266-230). New York: Harper \& Row.

Williams, G. C. \& Deci, E. (1996). Internalization of biopsychosocial values by medical students: A test of self-determination theory. Journal of Personality and Social Psychology, 70(4), 767-779.

Sobre as autoras

Sueli Edi Rufini Guimarães é Psicóloga, Mestre em Educação pela Universidade Estadual de Londrina e Doutora em Educação pela Universidade Estadual de Campinas. É Professora da Universidade Estadual de Londrina.

Evely Boruchovitch é Psicóloga, Ph.D. em Educação pela University of Southern California/LA.

É Professora da Universidade Estadual de Campinas. 